\title{
COVID-19: more than a respiratory virus, an optical coherence tomography study
}

\author{
Esra Dag Seker (D) - Inci Elif Erbahceci Timur
}

Received: 23 January 2021 / Accepted: 9 July 2021 / Published online: 27 July 2021

(C) The Author(s), under exclusive licence to Springer Nature B.V. 2021

\begin{abstract}
Purpose The purpose of this study is to investigate anatomic and morphologic features of inner and outer retinal layers in patients recovered from COVID-19 with Spectral Domain Optical Coherence Tomography (SD-OCT), whether correlate with any symptoms during disease process.

Methods 32 patients recovered from COVID-19 and age- and gender-matched 36 healthy controls were included in this cross-sectional study. Ganglion cellinner plexiform layer, macular and peripapiller retinal nerve fiber layer (RNFL), inner nuclear layer (INL), outer nuclear layer (ONL), outer plexiform layer (OPL) and the outer retinal hyperreflective bands including external limiting membrane (ELM), ellipsoid zone (EZ) and interdigitation zone (IZ) were examined with SD-OCT. The differences of each retinal layers thickness among subgroup analysis of ocular pain and headache were also compared.
\end{abstract}

Supplementary Information The online version contains supplementary material available at https://doi.org/10.1007/ s10792-021-01952-5.

E. Dag Seker $(\bowtie)$

Department of Ophthalmology, Ankara City Hospital, Universiteler Distinct, 1604. Street, No: 9, 06800 Bilkent, Ankara, Turkey

e-mail: dr.esra.seker@gmail.com

I. E. Erbahceci Timur

Department of Ophthalmology, Ministry of Health Ankara

City Hospital, Ankara, Turkey
Results Macular RNFL of inner and outer nasal and outer inferior quadrants were thinner in COVID-19 patients compared to healthy control group ( $p=0.046, p=0.014$ and $p=0.016$, respectively). Thinning in outer superior quadrant of GCIPL and INL quadrants were detected in patients with headache ( $p=0.026$ and $p=0.01)$. Superonasal and inferotemporal sectors of pRNFL were thinner in patients with ocular pain compared to patients without ocular pain ( $p=0.024$ and $p=0.015$ ). Integrity of EZ, ELM and IZ was evaluated as continuous line and protected on each OCT scans.

Conclusion The study demonstrated convincing evidence that SARS-CoV-2 can affect the inner and outer retinal layers, with subclinical localized alterations, particularly in patients with headache and ocular pain symptoms during COVID-19 period.

Keywords SARS-CoV-2 - COVID-19 - Eye · Retina $\cdot$ Optic coherence tomography $\cdot$ Headache

\section{Introduction}

Novel coronavirus disease (COVID-19) caused by respiratory syndrome coronavirus 2 (SARS-CoV-2) agent, first reported in Wuhan, China, in December 2019 [1-3], along with the fast spread [4] to whole world was declared as pandemic by the World Health 
Organisation [5, 6]. Altough it is defined as a respiratory virus, since the angiotensin-converting enzyme II (ACE-2), which the agent attaches [3], is expressed widely in the human body, the disease has the potential to damage many tissues and organs.

As the pandemic period continues, the scientific publications pointing ocular involvement have been increased. Ocular involvement can be in different clinical presentations. At the beginning of the pandemic, the publications were mainly about the anterior segment involvement [7-9] of the disease and the discussion was whether the transmission is possible from ocular surface [10] and how the best protection should be [11]. Recent studies are evolved to represent retinal involvement of SARS-CoV-2 infection in the ongoing process.

Retina can be affected by viral infections either with direct cytopathic effect of the virus on retinal neurons as in the case of cytomegalovirus [12], or secondary with a damage to the microvasculature when the virus targets the vascular endothelium like in HIV retinopathy [13]. Furthermore, SARS-CoV-2 has been detected in the retina of the deceased patients with COVID-19 [14].

Prothrombotic state and immune-mediated reactions triggered by COVID-19 infection can lead to retinal damage by endothelial dysfunction, which can cause cotton wool spots, edema and retinal hemorrhage clinically $[15,16]$.

Spectral domain optical coherence tomography (SD-OCT) is a non-invasive imaging technique that is useful for demonstrating subclinical retinal changes in systemic conditions such as diabetes mellitus $[17,18]$ and Alzheimer's disease [19, 20], and viral infections as well [21, 22].

The aim of this study is to investigate anatomic and morphologic features of inner and outer retinal layers with OCT in patients, recovered from COVID-19 with OCT, whether correlate with any symptom during disease process.

\section{Material and methods}

This study was approved by the Yildirim Beyazit University Medical School Committee of Clinical Trials and Ethics (Ankara, Turkey). All research procedures were performed in accordance with the Declaration of Helsinki. Written informed consent was obtained from the subjects with local institutional review board requirements. Between November and December 2020, patients recovered from COVID-19 were assessed by spectral domain optical coherence tomography (SD-OCT) for evaluating thickness of each retinal layers and peripapiller nerve fiber layers and by color fundus photographs in retina department of Ankara City Hospital, a tertiary referral education and research hospital, Ankara, Turkey. Consecutive 32 patients and age- and gender-matched 36 healthy controls were recruited in this cross-sectional study. All patients were healthcare professionals, recovered from confirmed COVID-19, either by showing viral genome in nasopharyngeal swab by reverse transcriptase polymerase chain reaction (RT-PCR), or by an antibody test of SARS-CoV-2 infection. Patients with any systemic or ocular diseases were excluded from the study. Also more than \pm 3 diopter spherical equivalent of refractive errors and use of any topical medication within last three months were exclusion criteria of the study.

Demographic and anamnestic data were collected by the same physician performing the examination. Each subject underwent a detailed ophthalmological examination including anterior segment and fundus slit lamp examination.

Optical coherence tomography

The SD-OCT (Heidelberg Engineering, Heidelberg, Germany) images were performed by an experienced technician, and OCT scans were assessed by two experienced clinicians independently without informed about study group of participants. Total retinal thickness was measured by using Macula Map $\mathrm{X}-\mathrm{Y}$ scanning protocol. The Macula Map scan pattern evaluates $6 \times 6 \mathrm{~mm}$ area centered on the fovea. Thickness of macular ganglion cell-inner plexiform layer (GCIPL) complex, macular retinal nerve fiber layer (RNFL), inner nuclear layer (INL), outer plexiform layer (OPL), and outer nuclear layer (ONL) was segmented automatically and measured by the caliper tool embedded in SD-OCT system (Fig. 1a). These layers were studied in three concentric rings centered in the fovea: central (1 mm), inner ring (1-3 mm) and outer ring (3-6 mm) which were determined by the Early Treatment Diabetic Retinopathy Study (ETDRS) [23]. 


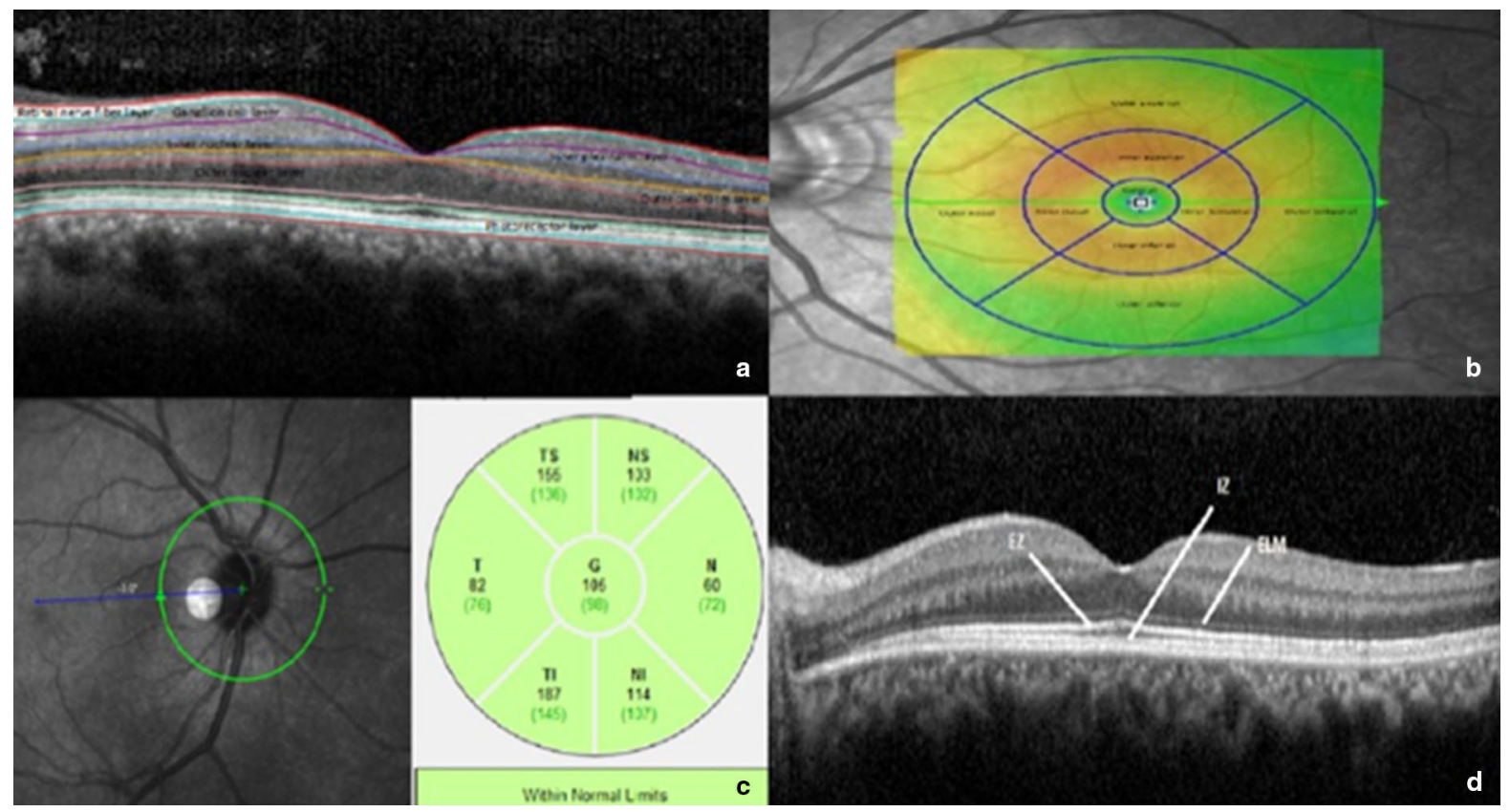

Fig. 1 a. A cross-sectional view of macula SD-OCT scan demonstrating the macular layer segmentation included in the study. b. ETDRS grid; the central circle is with a diameter of $1 \mathrm{~mm}$. The inner ring consists of the four quadrants surrounding the central circle with a diameter of $1-3 \mathrm{~mm}$. The outer ring consists of the four quadrants surrounding the inner ring, with a

Inner and outer rings were segmented into four quadrants (inner/outer superior, inner/outer inferior, inner/outer nasal, and inner/outer temporal) (Fig. 1b).

Peripapiller retinal nerve fiber layer ( $\mathrm{pRNFL}$ ) thickness was obtained from a circular scan with a diameter of $3.4 \mathrm{~mm}$ positioning on middle of the optic disc center. pRNFL was automatically segmented as central, temporal, inferotemporal, superotemporal, nasal, superonasal, and inferonasal quadrants (Fig. 1c).

The three hyperreflective outer retinal bands external limitan membrane (ELM), ellipsoid zone (EZ) and interdigitation zone (IZ) were identified according to the classification by International Nomenclature for Optical Coherence Tomography panel [24].

The disruption of ELM, IZ, and EZ was determined as the loss of the continuous back-reflection line that characterizes each layer. The disruption of these layers was interpreted in the central $1 \mathrm{~mm}$ of the three consecutive horizontal scans, and the median scan was located in the fovea. The disruption of the foveal ELM, $\mathrm{EZ}$, and IZ was graded as line not visible, disrupted in diameter of 3-6 mm. c. Analysis of peripapillary RNFL thickness; thickness of all quadrants was included in the study. d. Healthy OCT scan of three hyperreflective bands analyzed in the study. Early Treatment of Diabetic Retinopathy Study: ETDRS, ELM: external limiting membrane, EZ: ellipsoid zone, IZ: interdigitation zone

at least one scan (band defect) and continuous line (intact band) (Fig. 1d).

\section{Statistical analysis}

All analyses and calculations were performed via IBM SPSS Statistics 22.0 (IBM Corp. Released 2012. IBM SPSS Statistics for Windows, version 22.0. Armonk, NY: IBM Corp.). The distributions of the continuous variables in subgroups were examined by ShapiroWilk's test and normality plots. OCT measurements were summarized by mean \pm standard deviation (mean $\pm \mathrm{sd}$ ). Categorical variables were reported as frequency (\%), and comparison of categorical variables between groups was determined by using Chisquared test. The means of OCT measurements between groups were compared by independentsample $\mathrm{t}$ test or Mann-Whitney $U$ test. A $p$ value $<0.05$ was considered as statistically significant. 


\section{Results}

A total of 32 patients were included in the study, 14 (43.8\%) male, with mean age of $36.72+-9.98$ years. None of the patients required hospitalization, and only two patients $(6.2 \%)$ had pulmonary involvement. None of the patients had any systemic or ocular comorbidities. The mean time between confirmatory examination of COVID-19 (positive RT-PCR test for SARS-CoV-2) and eye examination was $60.5 \pm 38.6$ days.

The control group consisted of 36 healthy individuals mean age of $36.47+-9.98,12(33.4 \%)$ of male. During the acute phase of the infection, main ocular symptom was ocular pain, 12 of the patients $(37.5 \%)$ suffering from. Thickness of outer temporal quadrant of macular RNFL was $17.58 \pm 1.37 \mu \mathrm{m}$ in patients with ocular pain and $18.8 \pm 1.19 \mu \mathrm{m}$ in patients without ocular pain and thinner in patients with ocular pain $(p=0.009)$. Superonasal and inferotemporal sectors of pRNFL were $94.75 \pm 15.88 \mu \mathrm{m}$ and $143.9 \pm 14.85 \mu \mathrm{m}$ in patients with ocular pain; $108.2 \pm 13.84 \mu \mathrm{m}$ and $158.83 \pm 20.78 \mu \mathrm{m} \quad$ in patients without ocular pain and decreased in patients with ocular pain ( $p=0.024$ an $p=0.015)$.

Most frequent symptoms were fatigue $(81.3 \%)$ and myalgia $(81.3 \%)$ in 26 patients, athralgia $(71.9 \%)$ and anosmia $(71.9 \%)$ in 23 patients and headache in 20 patients $(62.5 \%)$. Clinical features of patients recovered from COVID-19 are shown in Table 1. Outer superior quadrants of GCIPL and INL were thinner, $68.85 \pm 9.28 \mu \mathrm{m}$ and $34.45 \pm 3.34 \mu \mathrm{m}$ in patients with headache; $\quad 64.33 \pm 5.44 \mu \mathrm{m} \quad$ and $32.42 \pm 2.27 \mu \mathrm{m}$ in patients without headache $(p=0.026$ and $p=0.01)$.

There were no anterior or posterior segment findings in patients. Evaluating color fundus photographs of each subjects to detect subtle or apparent retinal findings, no central or peripheral retinal abnormalities were observed. There was no difference in all quadrants of total retinal thickness between groups ( $p>0.05$, in each comparison). Macular retina nerve fiber layer of inner and outer nasal and outer inferior quadrants was thinner in COVID-19 patients compared to healthy control group $(p=0.046$, $p=0.014$, and $p=0.016$, respectively).

Inner temporal quadrant of GCIPL was $85.39 \pm 10.74 \mu \mathrm{m}$, thinner in patients and $89.18 \pm 7.67 \mu \mathrm{m}$ in healthy controls, and the differences was significant $(p=0.018)$. Central thickness of INL, ONL, and pRNFL was significantly thinner in patients recovered from COVID-19 when compared to healthy controls $(p=0.031, p=0.007$ and $p=0.013$, respectively). Reduced thickness of inner nasal quadrants of both OPL and ONL and outer nasal quadrant of OPL was determined in patients compared to controls $(p=0.004, p=0.004$ and $p=0.017$, respectively). There was no difference in all quadrants of total retinal thickness between groups ( $p>0.05$, in each comparison). Comparison of iner and outer retinal layers between patients recovered form COVID-19 and healthy controls by OCT is shown in Table 2.

There was no OCT scan completely or incompletely invisible of ELM, IZ, and EZ; therefore hyperreflective bands were evaluated as continuous line and protected on each OCT scans.

\section{Discussion}

Retinal findings of COVID-19 infection are of interest. Marinho et al. reported focal hyperreflective areas in the inner retina and also subtle cotton wool spots in a small amount patients with confirmed COVID-19 infection [25]. On the other hand, Vasvas et al. submitted that the hyperreflective areas on OCT scans could represent normal retinal vessels and cotton wool spots could represent myelinated nerve fiber layer or might be related to other retinal pathologies [26]. Subsequently, a case of papillophlebitis [27] and two cases of paracentral acute middle maculopathy and acute macular neuroretinopathy [28] were reported.

A study has reported flame-shaped hemorrhages and cotton wool spots in hospitalized severe COVID19 patients [29], and another study has reported cotton wool spots in patients hospitalized for COVID-19 pneumonia [30].

These studies showed that coronaviruses might induce various retinal pathologies. In this study, color fundus photographs of each subjects were taken to evaluate retinal abnormalities. Nevertheless, in our study we observed no subtle or apparent central or peripheral retinal findings such as vascular abnormalities or cotton wool spots determined by color fundus photographs or OCT scans. In many studies, ophthalmologic manifestations and retinal findings are reported to be related to severity of COVID-19 
Table 1 Clinical features of patients recovered from COVID-19

$$
(\mathrm{n}, \% / \text { mean } \pm \mathrm{SD})
$$

Patients recovered from COVID-19 (32)

\begin{tabular}{ll}
\hline Days since symptoms onset & $60.5 \pm 38.6$ \\
Duration of PCR + time (day) & $11.03 \pm 0.6$ \\
Ocular pain $-/+$ & $20(62.5 \%) / 12(37.5 \%)$ \\
Headache $-/+$ & $12(37.5 \%) / 20(62.5 \%)$ \\
Fever $-/+$ & $23(71.9 \%) / 9(28.1 \%)$ \\
Cough $-/+$ & $22(68.8 \%) / 10(31.2 \%)$ \\
Dyspne $-/+$ & $25(78.1 \%) / 7(21.9 \%)$ \\
Fatigue $-/+$ & $6(18.8 \%) / 26(81.3 \%)$ \\
Myalgia-/+ & $6(18.8 \%) / 26(81.3 \%)$ \\
Arthralgia $-/+$ & $9(28.2 \%) / 23(71.9 \%)$ \\
Anosmi $-/+$ & $9(28.1 \%) / 23(71.9 \%)$ \\
Agesuia $-/+$ & $10(31.3 \%) / 22(68.7 \%)$ \\
Involvement of lung $-/+$ & $30(93.8 \%) / 2(6.2 \%)$ \\
Treatment agent & \\
No treatment & $2(6.3 \%)$ \\
Favipiravir & $12(37.5 \%)$ \\
Hydroxychloroquine & $12(37.5 \%)$ \\
Favipiravir and hydroxychloroquine & $6(18.8 \%)$ \\
\hline
\end{tabular}

infection [29-32]. Nevertheless, retinal findings such as hemorrhages, cotton wool spots and vascular changes seem to be time dependent [31, 32]. Clinical features of patients recruited in this study, such as longer duration of post-COVID period until capturing OCT images, no requirement of hospitalization during COVID period, or relatively mild to moderate symptoms of COVID, might reflect the severity of disease, and less severe COVID-19 infection of our cases might explain the invisibility of these retinal findings.

ACE-2 is a cell entry receptor for SARS-CoV-2 to initiate inflammation and pathologic processes [3]. ACE-2 has been detected in human neural retina [33], ganglion cells, cells of inner nuclear layer, photoreceptors [34], and vascularized retinal pigment epithelium and choroid [35]. Therefore, alterations in these various retinal layers might be expected during COVID period. In this study, SD-OCT was used to compare the thickness of each retinal layer of patients recovered from COVID-19 infection with age- and gender-matched healthy subjects and decrease in the localized retinal thickness was determined. Thinning in inner temporal quadrant of GCIPL thickness and central quadrant of INL, ONL and pRNFL thickness was detected.
After reports of microvascular injury and thrombosis in the pathogenesis of severe COVID-19 infection [36, 37], optical coherence tomography angiography (OCTA) analysis was performed in recovered COVID-19 patients versus age-matched controls to evaluate retinal vascular involvement. Abrishami et al. determined reduced vessel density in superficial and deep capillary plexus of the foveal and parafoveal regions regardless of hospitalization [38], and Savastano et al. identified reduced perfusion density of the radial peripapillary capillary plexus (RPCP) [39]. Reduction in RPCP reflects the impairment of homeostasis and function of retinal ganglion cells and their axons that is correlated to thinning of RNFL thickness in glaucoma patients [40]. Thinning of inner temporal quadrant of GCIPL, central pRNFL and inner nasal, outer nasal and inferior quadrants of macular RNFL thickness in patients recovered from COVID-19 compared with healthy subjects was detected in this study. The localized subclinical axonal damage might be related to reduction in RPCP. Ornek et al. also demonstrated localized thinning of pRNFL in patients with COVID-19 [41] which was consistent with our study.

To the best of our knowledge, there have been no studies published on determining structural properties 
Table 2 Comparison of inner and outer retinal layers between patients recovered from COVID-19 and healthy controls

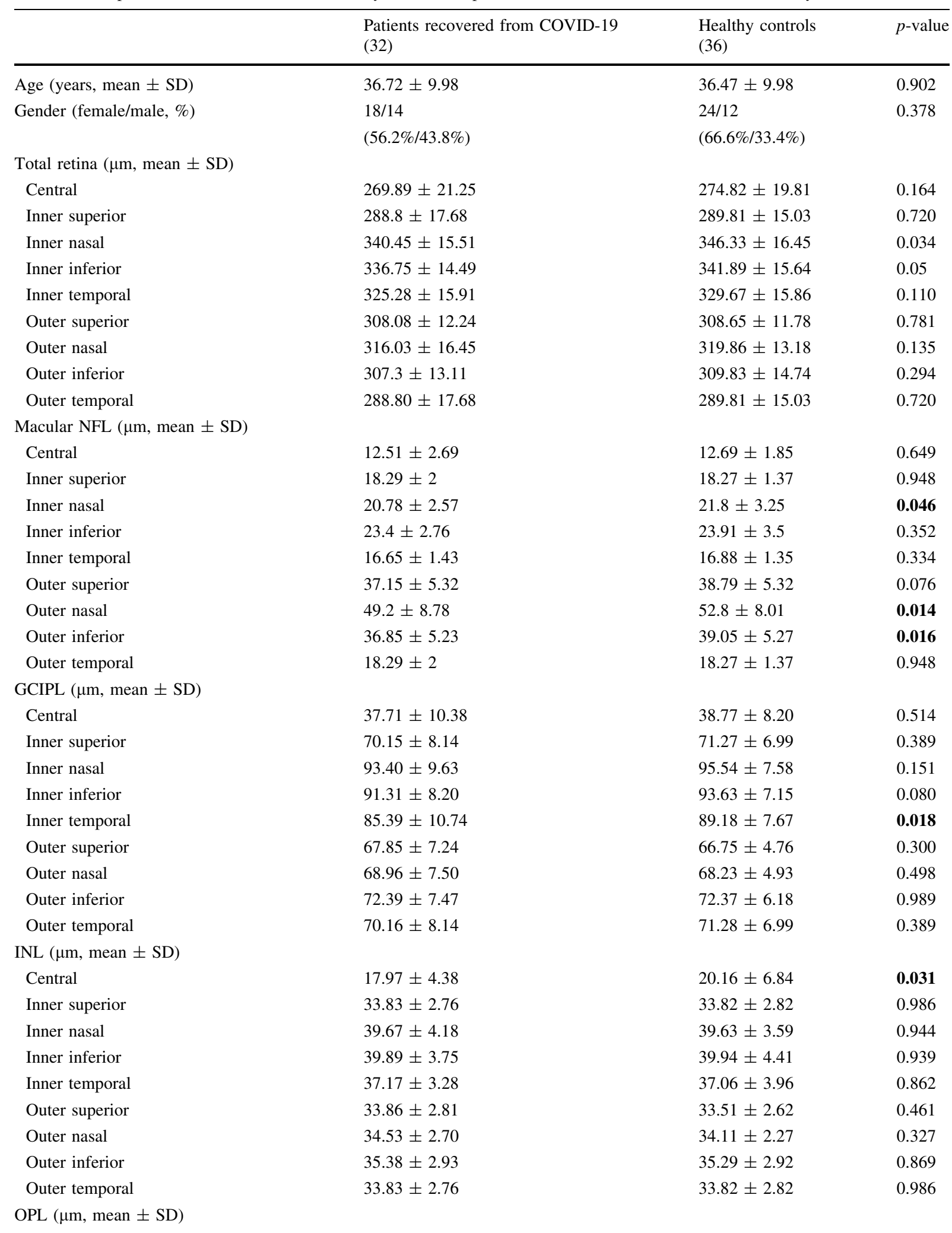


Table 2 continued

\begin{tabular}{|c|c|c|c|}
\hline & $\begin{array}{l}\text { Patients recovered from COVID-19 } \\
\text { (32) }\end{array}$ & $\begin{array}{l}\text { Healthy controls } \\
\text { (36) }\end{array}$ & $p$-value \\
\hline Central & $27.14 \pm 7.87$ & $24.93 \pm 5.41$ & 0.062 \\
\hline Inner superior & $27.31 \pm 2.29$ & $27.72 \pm 7.11$ & 0.660 \\
\hline Inner nasal & $30.03 \pm 4.21$ & $32.70 \pm 6.28$ & 0.004 \\
\hline Inner inferior & $34.64 \pm 8.55$ & $33.10 \pm 8.36$ & 0.290 \\
\hline Inner temporal & $30.41 \pm 3.89$ & $30.28 \pm 3.78$ & 0.846 \\
\hline Outer superior & $28.02 \pm 3.84$ & $27.61 \pm 3.18$ & 0.504 \\
\hline Outer nasal & $27.46 \pm 2.34$ & $28.53 \pm 2.82$ & 0.017 \\
\hline Outer inferior & $29.16 \pm 4.30$ & $28.19 \pm 3.24$ & 0.141 \\
\hline Outer temporal & $27.31 \pm 2.29$ & $27.72 \pm 7.11$ & 0.660 \\
\hline \multicolumn{4}{|c|}{ ONL $(\mu \mathrm{m}$, mean $\pm \mathrm{SD})$} \\
\hline Central & $87.38 \pm 11.45$ & $93.31 \pm 13.60$ & 0.007 \\
\hline Inner superior & $58.11 \pm 7.39$ & $60.33 \pm 7.45$ & 0.084 \\
\hline Inner nasal & $72.67 \pm 9.96$ & $77.61 \pm 9.85$ & 0.004 \\
\hline Inner inferior & $66.30 \pm 11.46$ & $69.88 \pm 11.98$ & 0.078 \\
\hline Inner temporal & $72.55 \pm 7.08$ & $75.15 \pm 9.08$ & 0.063 \\
\hline Outer superior & $62.09 \pm 8.13$ & $62.67 \pm 8.08$ & 0.681 \\
\hline Outer nasal & $58.69 \pm 7.82$ & $60.17 \pm 7.38$ & 0.259 \\
\hline Outer inferior & $56.39 \pm 7.90$ & $58.04 \pm 7.56$ & 0.216 \\
\hline Outer temporal & $58.11 \pm 7.39$ & $60.33 \pm 7.45$ & 0.084 \\
\hline \multicolumn{4}{|c|}{ Peripapiller RNFL $(\mu \mathrm{m}$, mean \pm SD) } \\
\hline Central & $99.56 \pm 8.68$ & $104.19 \pm 12.18$ & 0.013 \\
\hline Superotemporal & $135.50 \pm 20.34$ & $138.92 \pm 15.85$ & 0.281 \\
\hline Superonasal & $71.78 \pm 11.38$ & $75.24 \pm 11.32$ & 0.079 \\
\hline Nasal & $72.17 \pm 14.36$ & $76.07 \pm 19.50$ & 0.191 \\
\hline Inferonasal & $114.56 \pm 21.06$ & $119.24 \pm 22.87$ & 0.219 \\
\hline Inferotemporal & $147.44 \pm 18.88$ & $148.54 \pm 18.80$ & 0.734 \\
\hline Temporal & $781.78 \pm 11.38$ & $75.24 \pm 11.32$ & 0.079 \\
\hline
\end{tabular}

GCIPL Ganglion cell-inner plexiform layer, INL İnner nuclear layer, $N F L$ Nerve fiber layer, $O N L$ Outer nuclear layer, $O P L$ Outer plexiform layer, $R N F L$ Retinal nerve fiber layer

of outer retinal hyperreflective bands and qualitative analysis of outer retinal layers in patients recovered from COVID-19 compared with healthy control subjects. Defects in outer retinal layers were described as case reports with acute macular neuroretinitis, paracentral acute middle maculopathy, or outer retinal band abnormalities [28, 42]. In our study, there was no disruption in integrity of external limitan membrane, ellipsoid zone, or interdigitation zone, but localized subclinical thinning in outer retinal layers was prominent in inner nasal quadrant of both outer plexiform and outer nuclear layers; thinning in outer nasal quadrant of OPL and central quadrant of ONL was also determined.

Thinning of GCIPL and more prominent outer nuclear layer could be in relation to neuroinvasive potential of COVID-19 as central nervous system manifestation [43], described in animal studies [44] or neurologic events associated with SARS-CoV-2 [45]. In an animal study on the murine coronavirus mouse hepatitis virus strain, effects in different retinal layers were demonstrated [46], and virus antigens in inner 
and outer retinal layers were detected in another study [47]. Vulnerability of inner layers of retina especially GCIPL rather than outer layers of retina [48] and also decreased vessel density of superficial and deep capillary plexus [38] after COVID-19 infection might explain the alterations of thickness to be more prominent in inner retinal layers and preserved outer retinal bands.

Neurotropism of the virus has been proposed as one of the mechanisms for the neurological and neuroophthalmic manifestations [49]. A prominent tropism to neurosensorial retinal layers regardless of inoculation route was observed in murine coronavirus, and gliosis might be seen in affected retinal layers as a result of damage in blood-ocular barrier [46, 50]. Eye seems to be crucial in understanding pathophysiology of SARS-CoV-2 and should be emphasized more [51].

As the COVID-19 pandemic progresses, reports of neurological manifestations are increasing [52, 53]. Headache is the most common neurological symptom of COVID-19 and could be due to direct invasion of SARS-CoV-2 or stimulated trigeminal nerve endings with pro-inflammatory mediators and cytokines in the nasal cavity or trigemino-vascular activation with involvement of the vascular endothelial cells [54]. In our study, outer superior quadrant of GCIPL and INL thickness was reduced in patients with headache $(62.5 \%)$ compared to patients without headache. Ocular pain was the most frequent ocular symptom in our study, 12 patients (37\%) suffering from. This pain was described as a pressure on both eyes. We analyzed that the patients with ocular pain, outer temporal quadrant of macular RNFL, and superonasal and inferotemporal sectors of pRNFL were thinner compared to patients without ocular pain. Although headache and ocular pain are known as nonspecific neurologic symptoms, we determined retinal thickness in subgroup analysis of patients with these symptoms, pointing the subclinic localized damage in macular and peripapiller RNFL, that could regard to neurological involvement and those patients should be followed up closely.

Patients enrolled in the study were with mild to moderate COVID symptoms and without visual complaints; long duration after COVID recovery for ophthalmic evaluation, small sample size and limited age range were limitations of this study. Also evaluating retinal vasculature parameters with an accurate imaging method such as OCT-angiography and evaluating function of outer retinal layer with electrophysiological tests were lack in this study.

In conclusion, this study demonstrated convincing evidence that SARS-CoV-2 can affect the inner and outer retinal layers, with subclinical localized alterations and preserved integrity of outer retinal hyperreflective bands. Retinal imaging by optical coherence tomography is a non-invasive, reproducible, and expeditious technique that subclinic or apparent retinal pathologies might be detected during COVID-19 period. Management of COVID-19 patients should include retinal assessment, with a close follow-up, especially in patients with headache and ocular pain. The results of this study could highlight pathophysiology of COVID-19, especially in ocular involvement with neurological symptoms. Future studies are needed to evaluate whether these alterations of COVID-19 at retinal layers have permanent and long-term effects.

Acknowledgements The authors thank Professor Nagihan Uğurlu, MD, $\mathrm{PhD}$, for providing general support.

Author contribution Both authors contributed to the study design. Data collection, interpretation, analysis, and writing manuscript were performed together. Both authors read and approved the final manuscript.

Funding No funds, grants, or other support was received for conducting this study and preparing this manuscript.

Availability of data and material Supplemental materials for this article are available from the corresponding author on reasonable request.

\section{Declarations}

Conflict of interest The authors declare that they do not have any conflict of interest.

Ethical approval Approval was obtained from the Ethics Committee of Yildirim Beyazit University, Ankara, Turkey. All procedures performed in the study involving human participants were in accordance with the ethical standards and also with Declaration of Helsinki.

Informed consent Informed consent was obtained from all individual participants included in the study.

Consent for publication Both authors have agreed to the submission fort this article. 


\section{References}

1. Lu H, Stratton CW, Tang YW (2020) Outbreak of pneumonia of unknown etiology in Wuhan China: the mystery and the miracle. J Med Virol 92(4):401-402

2. Du Toit A (2020) Outbreak of a novel coronavirus. Nat Rev Microbiol 18(3):123

3. Zhou P, Yang XL, Wang XG, Hu B, Zhang L, Zhang W et al (2020) A pneumonia outbreak associated with a new coronavirus of probable bat origin. Nature 579:270-273

4. Bogoch II, Watts A, Thomas-Bachli A, Huber C, Kraemer MUG, Khan K (2020) Pneumonia of unknown aetiology in Wuhan, China: potential for international spread via commercial air travel. J Trav Med. https://doi.org/10.1093/jtm/ taaa008

5. Jee Y (2020) WHO International Health Regulations Emergency Committee for the COVID-19 outbreak. Epidemiol Health 42:e2020013

6. Cucinotta D, Vanelli M (2020) WHO Declares COVID-19 a Pandemic. Acta Biomed 91(1):157-160

7. Wu P, Duan F, Luo C, Liu Q, Qu X, Liang L et al (2020) Characteristics of ocular findings of patients with coronavirus disease 2019 (COVID-19) in Hubei Province. China, JAMA Ophthalmol 138(5):575-578

8. Khavandi S, Tabibzadeh E, Naderan M, Shoar S (2020) Corona virus disease-19 (COVID-19) presenting as conjunctivitis: atypically high-risk during a pandemic. Cont Lens Anterior Eye 43(3):211-212

9. Chen L, Deng C, Chen X, Zhang X, Chen B, Yu H et al (2020) Ocular manifestations and clinical characteristics of 535 cases of COVID-19 in Wuhan, China: a cross-sectional study. Acta Ophthalmol 10(1111):14472

10. Lu CW, Liu XF, Jia ZF (2020) 2019-nCoV transmission through the ocular surface must not be ignored. The Lancet 395(10224):39

11. Li JO, Lam DSC, Chen Y, Ting DSW (2020) Novel coronavirus disease 2019 (COVID-19): the importance of recognising possible early ocular manifestation and using protective eyewear. Br J Ophthalmol 104(3):297-298

12. Port AD, Orlin A, Kiss S, Patel S, D'Amico DJ, Gupta MP (2017) Cytomegalovirus retinitis: a review. J Ocul Pharmacol Ther 33(4):224-234

13. Agarwal A, Invernizzi A, Acquistapace A (2017) Analysis of retinochoroidal vasculature in human immunodeficiency virus infection using spectral-domain OCT angiography. Ophthalmol Retina 1(6):545-554

14. Casagrande M, Fitzek A, Püschel K, Aleshcheva G, Schultheiss HP, Berneking L et al (2020) Detection of SARS-CoV-2 in human retinal biopsies of deceased COVID-19 patients. Ocul Immunol Inflamm 28(5):721-725

15. Gavriilaki E, Brodsky RA (2020) Severe COVID-19 infection and thrombotic microangiopathy: success does not come easily. Br J Haematol 189(6):e227-e230

16. D'Aloisio R, Nasillo V, Gironi M, Mastropasqua R (2020) Bilateral macular hemorrhage in a patient with COVID-19. Am J Ophthalmol Case Rep. 20:100958

17. Mendez N, Kommana SS, Szirth B, Khouri AS (2015) Structural Changes by Spectral Domain Optical Coherence Tomography in Patients With Type 1 Diabetes Mellitus. J Diabetes Sci Technol 10(2):271-276
18. Saxena S, Caprnda M, Ruia S, Prasad S, Ankita FJ et al (2019) Spectral domain optical coherence tomography based imaging biomarkers for diabetic retinopathy. Endocrine 66(3):509-516

19. Mutlu U, Colijn JM, Ikram MA, Bonnemaijer PWM, Licher S, Wolters FJ et al (2018) Association of retinal neurodegeneration on optical coherence tomography with dementia: a population-based study. JAMA Neurol 75(10):1256-1263

20. Coppola G, Di Renzo A, Ziccardi L, Martelli F, Fadda A, Manni G et al (2015) Optical coherence tomography in alzheimer's disease: a meta-analysis. PLoS ONE 10(8):e0134750. https://doi.org/10.1371/journal.pone. 0134750.PMC4529274

21. Kurup SP, Khan S, Gill MK (2014) Spectral domain optical coherence tomography in the evaluation and management of infectious retinitis. Retina 34(11):2233-2241

22. Invernizzi A, Agarwal A, Ravera V, Oldani M, Staurenghi G, Viola F (2018) Optical coherence tomography findings in cytomegalovirus retinitis: a longitudinal study. Retina 38(1):108-117

23. Grading diabetic retinopathy from stereoscopic color fundus photographs-An extension of the modified Airlie House classification. ETDRS report number 10 (1991) Early treatment diabetic retinopathy study research group. Ophthalmology 98:86-806

24. Staurenghi G, Sadda S, Chakravarthy U et al (2014) Proposed lexicon for anatomic landmarks in normal posterior segment spectral-domain optical coherence tomography. Ophthalmology 121:1572-1578

25. Marinho PM, Amarcos AA, Romano AC, Nascimento H, Belfort R Jr (2020) Retinal findings in patients with COVID-19. Lancet 395(10237):1610-1616

26. Vasvas DG, Sarraf D, Sadda SR, Eliott D, Ehlers JP, Waheed NK et al (2020) Concerns about the interpretation of OCT and fundus findings in COVID-19 patients in recent Lancet publication. Eye (london) 9:1-2

27. Insausti-García A, Reche-Sainz JA, Ruiz-Arranz C, López Vázquez Á, Ferro-Osuna M (2020) Papillophlebitis in a COVID-19 patient: inflammation and hypercoagulable state. Eur J Ophthalmol. https://doi.org/10.1177/ 1120672120947591

28. Virgo J, Mohamed M (2020) Paracentral acute middle maculopathy and acute macular neuroretinopathy following SARS-CoV-2 infection. Eye (London) 34(12):2352-2353. https://doi.org/10.1038/s41433-020-1069-8

29. Pereira LA, Soares LCM, Nascimento PA, Cirillo LRN, Sakuma HT, Veiga GLD et al (2020) Retinal findings in hospitalised patients with severe COVID-19. Br J Ophthalmol. https://doi.org/10.1136/bjophthalmol-2020317576

30. Landecho MF, Yuste JR, Gándara E, Sunsundegui P, Quiroga J, Alcaide AB et al (2020) COVID-19 retinal microangiopathy as an in vivo biomarker of systemic vascular disease? J Intern Med. https://doi.org/10.1111/joim. 13156

31. Invernizzi A, Torre A, Parrulli S, Zicarelli F, Schiuma M, Colombo V et al (2020) Retinal findings in patients with COVID-19: Results from the SERPICO-19 study. E Clinical Medicine 27:100550

32. Zapata MA, Banderas Garcia S, Sanchez-Moltalva A, Falco A, Otero-Romero S, Arcos G et al (2020) Retinal 
microvascular abnormalities in patients after COVID-19 depending on disease severity. Br J Ophthalmol. https://doi. org/10.1136/bjophthalmol-2020-317953

33. Senanayake P, Drazba J, Shadrach K, Milsted A, RunggerBrrandle E, Nishiyama K et al (2007) Angiotensin II and its receptor subtypes in the human retina. Invest Ophthalmol Vis Sci 48:3301-3311

34. Savaskan E, Loffler K, Meier F, Müller-Spahn F, Flammer J, Meyer P, (2004) Immunohistochemical localization of angiotensin-converting enzyme, angiotensin II and angiotensin AT1 receptor in human ocular tissues. Ophthalmic Res 36:312-320

35. Wagner J, Jan Danser AH, Derkx FH, de Jong TV, Paul M, Mullins JJ et al (1996) Demonstration of renin mRNA, angiotensinogen mRNA, and angiotensin converting enzyme mRNA expression in the human eye: evidence for an intraocular renin-angiotensin system. Br J Ophthalmol 80:159-163

36. Magro C, Mulvey JJ, Berlin D, Nuovo G, Salvatore s, Harp J, et al (2020) Complement associated microvascular injury and thrombosis in the pathogenesis of severe COVID-19 infection: a report of five cases. Transl Res 220:1-13

37. Helms J, Tacquard C, Severac F (2020) High risk of thrombosis in patients with severe SARS-CoV-2 infection: a multicenter prospective cohort study. Intens Care Med. https://doi.org/10.1007/s00134-020-06062-x

38. Abrishami M, Emamverdian Z, Shoeibi N, Omidtabrizi A, Daneshvar R, Saeidi Rezvani T et al (2020) Optical coherence tomography angiography analysis of the retina in patients recovered from COVID-19: a case-control study. Can J Ophthalmol S0008-4182(20):30813-30819. https:// doi.org/10.1016/j.jcjo.2020.11.006

39. Savastano A, Crincoli E, Savastano MC, Gemelli Against Covid-Post-Acute Care Study Group (2020) Peripapillary retinal vascular involvement in early post-COVID-19 patients. J Clin Med 9:E2895

40. Mammo Z, Heisler M, Balaratnasingam C, Lee S, Yu D-Y, Schendel MP, S, et al (2016) Quantitative optical coherence tomography angiography of radial peripapillary capillaries in glaucoma, glaucoma suspect, and normal eyes. Am J Ophthalmol 170:41-49

41. Ornek K, Temel E, Asıkgarip N, Kocamis O (2020) Localized retinal nerve fiber layer defect in patients with COVID-19. Arq Bras Oftalmol 83(6):562-563

42. Zago Filho LA, Lima LH, Melo GB, Zett C, Farah ME (2020) Vitritis and outer retinal abnormalities in a patient with COVID-19. Ocul Immunol Inflamm. https://doi.org/ 10.1080/09273948.2020.1821898

43. Li YC, Bai WZ, Hashikawa T (2020) The neuroinvasive potential of SARS-CoV2 may play a role in the respiratory failure of COVID-19 patients. J Med Virol. https://doi.org/ 10.1002/jmv.25728

44. Wang Y, Detrick B, Yu ZX, Zhang J, Hooks CL, JJ (2000) The role of apoptosis within the retina of coronavirus-infected mice. Invest Ophthalmol Vis Sci 41(10):3011-3018

45. Abboud H, Abboud FZ, Kharbouch H, Arkha Y, Abbadi NE, Ouahabi AE (2020) COVID-19 and SARS-CoV-2 infection: pathophysiology and clinical effects on the nervous system. World Neurosurg 140:49-53

46. Robbins SG, Detrick B, Hooks JJ (1990) Retinopathy following intravitreal injection of mice with MHV strain JHM. Adv Exp Med Biol. https://doi.org/10.1007/978-1-46845823-7_72

47. Seah I, Agrawal R (2020) Can the coronavirus disease 2019 (COVID-19) affect the eyes? A review of coronaviruses and ocular implications in humans and animals. Ocul Immunol Inflamm 28(3):391-395. https://doi.org/10.1080/09273948. 2020.1738501

48. Kaur C, Foulds WS, Ling EA (2008) Hypoxia-ischemia and retinal ganglion cell damage. Clin Ophthalmol 2(4):879-889

49. Costello F, Dalakas MC (2020) Cranial neuropathies and COVID-19 Neurotropism and autoimmunity. Neurology 95(5):195-196

50. Neri P, Pichi F (2020) COVID-19 and the eye immunity: lesson learned from the past and possible new therapeutic insights. Int Ophthalmol 40(5):1057-1060. https://doi.org/ 10.1007/s10792-020-01389-2.PMID:32314322;PMCID: PMC7167536

51. Neri P, Lamperti M, Pichi F (2020) SARS-COV-2 and eye immunity: the lesson was learned but we are not done yet Brainstorming on possible pathophysiology inspired by ocular models. Int Ophthalmol 40(8):1879-1883

52. Ellul MA, Benjamin L, Singh B, Lant S, Michael BD, Easton A et al (2020) Neurological associations of COVID19. Lancet Neurol 19(9):767-783. https://doi.org/10.1016/ S1474-4422(20)30221-0

53. Bellocchio L, Bordea IR, Ballini A, Lorusso F, Hazballa D, Isacco CG et al (2020) Environmental issues and neurological manifestations associated with COVID-19 pandemic: new aspects of the disease? Int J Environ Res Public Health 17(21):8049. https://doi.org/10.3390/ ijerph17218049

54. Bolay H, Gül A, Baykan B (2020) COVID-19 is a real headache! Headache 60(7):1415-1421. https://doi.org/10. 1111/head.13856

Publisher's Note Springer Nature remains neutral with regard to jurisdictional claims in published maps and institutional affiliations. 